\title{
Classification of seizure and seizure free EEG signals using optimal mother wavelet and relative power
}

\author{
Nilima Salankar', Sangita B. Nemade ${ }^{2}$, Varsha P. Gaikwad ${ }^{3}$ \\ ${ }^{1}$ Department of Virtualization, University of Petroleum and Energy Studies, India \\ ${ }^{2,3}$ Department of Information Technology, Govt. College of Engineering Aurangabad, India
}

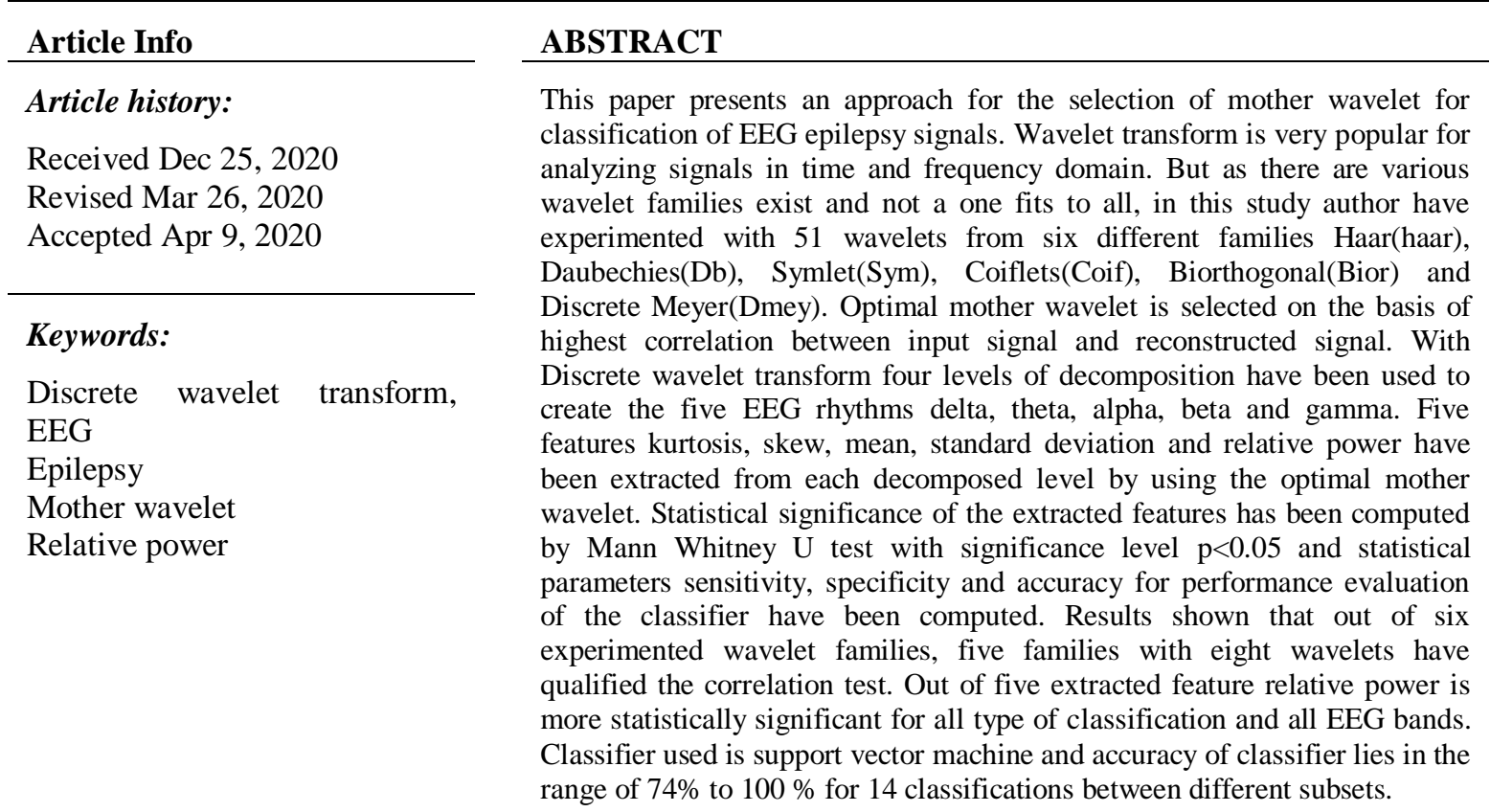

Copyright $\odot 2020$ Institute of Advanced Engineering and Science. All rights reserved.

\author{
Corresponding Author: \\ Nilima Salankar, \\ Department of Virtualization, \\ School of Computer Science, \\ University of Petroeum and EnergyStudies, Dehradun. \\ Email: nilima11123@gmail.com
}

\section{INTRODUCTION}

Neurological disorder, epilepsy is affecting $1 \%$ of the world's population[1] and about $30 \%$ of subject suffering from epilepsy are not benefited by medication [2,3], automatic, semiautomatic or visual careful analysis of the electroencephalogram [4] records can provide valuable, precise and clear insights into this widespread brain disorder. Base for the accurate diagnosis of disorder lies with accurate parameters retrieval from EEG signals and those parameters can be used as valuable diagnostic features for detection and discrimination of epileptic seizure. EEG signal can be considered as stationary or non-stationary depends upon the selection of window size and behaviour of data samples in the recording. Time domain as well as frequency domain features can be extracted from EEG signal to provide the classification between seizure and seizure free subjects. Both time domain and frequency domain features have been used for the classification of epileptic seizure from EEG signals, by assuming EEG signal exhibits stationarity [5, 6]. By assuming EEG signal exhibits non stationarity [7, 8] time and frequency methods have been developed. The various methods for analysis and classification of epileptic seizure EEG signals using the wavelet 
transform [9-15] and the multi wavelet transform [16] have been developed. An EEG signal is band limited in frequency $(0.1-60 \mathrm{~Hz})$, modelled and classified into five rhythmic waves, delta, theta, alpha, beta and gamma waves which are responsible to capture different associated brain activities inside the brain [17]. Correct diagnosis of epileptic seizure [18-21] demands precise feature extraction and accuracy in classification. Mere Visual inspection and analysis of EEG signal requires expertise in neurological domain [22] which turns this complete process very time consuming, tedious and subjective [23] and may lead to incorrect diagnosis. To overcome this limitations and provide timely diagnosis to sufferer either fully automated or semi-automated analysis of EEG signal is required [24]. Several processing techniques proposed and available in the literature assumes that the EEG is generated by a highly complex linear system, but functioning of brain is non-linear [25]. Discrete wavelet transform is very precise method to subdivide the EEG signals by retaining valuable time and frequency information. The proposed approach in this paper is mainly focus on signal dependent optimal mother wavelet selection on the basis of correlation factor between input signal and reconstructed signal for diagnosis of epilepsy, followed by feature extraction at each sub band level which helps the classifier to reach to the maximum level of accuracy. Classification has been done between 14 different subsets, by using support vector machine (SVM).

As per the best knowledge of an author, this is the first work that presents the approach for selection of signal dependent mother wavelet from orthogonal as well as bi-orthogonal family of wavelet and combination of relative power, kurtosis, skew, mean, standard deviation as features. This study has considered all the possible cases which may results into the seizure activity.

The rest of the paper is organized as follows: In Section 2 methodology has been discussed which describes dataset, discrete wavelet transform, optimal mother wavelet selection process, feature extraction and design of classifier. Section 3 providesresults which include computation of statistical significance of extracted feature and computation of statistical parameters sensitivity (SEN), Specificity (SPEC) and Accuracy (ACC) for performance evaluation of the classifier. Section 4 discusses about results. Conclusion has been drawn in Section 5.

\section{METHODOLOGY}

In this section details of the dataset used, DWT method adopted to decompose the signal has explained, approach used for the selection of optimal mother on the basis of correlation, feature extraction and model of the entire process is explained.

\subsection{Dataset}

The EEG dataset used in this study is as described in [26] is available online in public domain. This dataset has captured EEG signals from the subjects of two categories healthy and epileptic. Healthy subjects are further categorized as eyes open and eyes closed which has a significant impact on appearance and disappearance on alpha waves. For Epileptic subjects signals has captured for actual seizure activity and in absence of seizure activity. For non-seizure activity signals has captured from tumor area and non-tumor area. These entire recordings are available as five subsets denoted as $\mathrm{Z}, \mathrm{O}, \mathrm{N}, \mathrm{F}$ and $\mathrm{S}$; where $\mathrm{Z}$ and $\mathrm{O}$ is healthy subject with eyes open and eyes closed respectively and EEG signals measured from the surface. F, $\mathrm{N}$ and $\mathrm{S}$ are measured from the intracranial. Each subset contains 100 single channel EEG signals, with duration $23.6 \mathrm{sec}$ and the sampling rate of $173.61 \mathrm{~Hz}$. All EEG signals were recorded with the same 128 channel amplifier system with an average common reference. To check the strength of proposed approach and to validate the performance of the classifier the EEG signals are categorized into 14 different classes, Eyes Open $(\mathrm{Z})$ vs. Eyes Closed(O), Eyes Open $(\mathrm{Z})$ vs. Seizure Free(F,N), Eyes Open $(\mathrm{Z})$ vs. Seizure Free tumour free area(F),Eyes Open $(Z)$ vs. Seizure Free tumour area(N), Eyes Open $(Z)$ vs. Ictal $(S)$, Eyes Closed(O) vs. Seizure Free(F,N),Eyes Closed(O) vs. Seizure Free tumour free area(F),Eyes Closed(O) vs. Seizure free tumour area(N),Eyes $\operatorname{Closed}(\mathrm{O})$ vs. Ictal(S), Healthy(Z,O) vs. Seizure free(F,N), Seizure free tumour free Area (F) vs. Seizure free tumour Area(N), Seizure free tumour free Area $(F)$ vs. Seizure(S), Seizure free tumor Area $(N)$ vs. Seizure(S), Seizure(F,N) vs. Seizure (S).

\subsection{Discrete wavelet transform}

Wavelet Transform is like a microscope to view the details of signal and it has very broad application area in various engineering, biomedical, weather forecast for solving various real life problems. To view the details of the signal various methods are available like Fourier transform (FT), Short time Fourier transform (STFT). Fourier transform of a signal extract the frequency contents of the signal over analysis window but it lacks to provide any information related to time of occurrence of frequency content and thus suitable for non-stationary signal where assumption is all the frequency available all the time. But if one needs to deal with non-stationary signals then fourier transform is not an option to handle the signal. 
Other alternative is STFT, which is in between compromised solution to provide the details of signal in frequency domain as well as time domain, but window size used for the analysis is same irrespective of high and low frequency content, thus frequency resolution is fixed for the entire length of signal. Thus even though STFT provides information of time and frequency it's not an optimal solution to view detailing of signal with varying time and frequency resolution as per requirement of study of biological signals. Unlike STFT wavelet transform(WT) provides a more flexible way of time and frequency representation of the signal as wavelet make use of flexible window size as per the frequency contents. Its make use of low scale for high frequencycontents and high scale for low frequency contents and thus window size is dynamic in nature. Window size in STFT is of fixed nature and window size of WT is of dynamic nature but in both the cases area of window remains the same. Thus WT gives precious frequency information at low frequency and precious time information for high frequencywhich is required for detailanalysis of the signal.

Levels of decomposition depend upon the original sampling frequency of the signal. At every decomposition level original signal pass through the pair of LP and HP named as quadrature mirror filters, thus signal is decomposed into two parts. The LP is the scaling function and HP is the wavelet function. The outputs from the low pass filter and high pass filter are known as approximate coefficient (CA) and detail coefficient (CD) respectively. At each decomposed level the output signals have half the frequency bandwidth of the original signal can be down sampled by two according to Nyquist rule. Each step of this decomposition process work to halved the time resolution and doubled the frequency resolution. Downsampling by 2 supports to halve the time resolution and filter supports to double the frequency resolution. The detail diagram for the four level decomposition of EEG signal with original sampling frequency by using DWT is as shown in Figure 1 The sampling frequency (fs) of original EEG signal in this study is 173.6 $\mathrm{Hz}$. As per the Nyquist frequency fs $/ 2=86.80 \mathrm{~Hz}$ has been considered. The computation of the co-efficient CA1 $=0$ to $\mathrm{fs} / 2 ; \mathrm{CD} 1=\mathrm{fs} / 2$ to fs; $\mathrm{CA} 2=0$ to $\mathrm{fs} / 4 ; \mathrm{CD} 2=\mathrm{fs} / 4$ to $\mathrm{fs} / 2 ; \mathrm{CA} 3=0$ to fs/8; $\mathrm{CD} 3=\mathrm{fs} / 8$ to fs $/ 4 ; \mathrm{CA} 4=0$ to $\mathrm{fs} / 16 ; \mathrm{CD} 4=\mathrm{fs} / 16$ to $\mathrm{fs} / 8$, where fs is the sampling frequency of the signal $\mathrm{x}[\mathrm{n}]$.

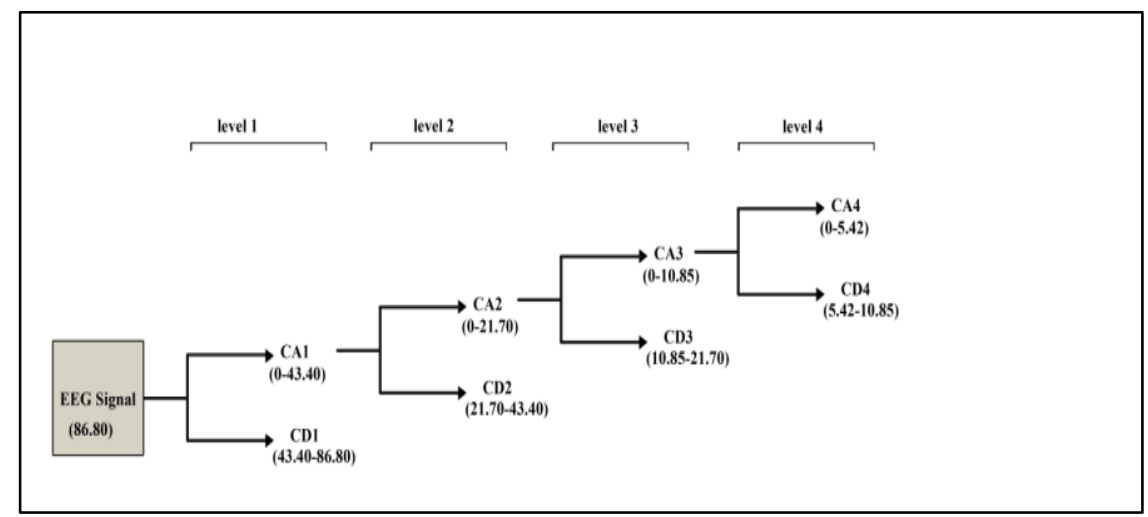

Figure 1. Discrete wavelet transform four level of decomposition

\subsection{Optimal mother wavelet selection}

Even though WT is the best choice to visualize details of the signal in time as well as frequency domain. Best performance of the wavelet lies in the selection of appropriate mother wavelet (MWT). There are many types of wavelet families are available which share some common properties. Accurate MWT selection not only helps to retain the original contents of the signal but also gives precious insights of frequency information and time information. Selection of mother wavelet can be done by visual inspection of the signal and wavelet function, which is very tedious job. In this paper authors have proposed an approach for selection of mother wavelet on the basis of correlation factor. In any type of transformation main objective is to reduce the artifacts which contaminate the signal. Experimentation with 51 wavelets has been carried out, details of wavelet family. In literature the mostly reported wavelet used belong to orthogonal families such as Db, Coif and Sym help to conserve decomposed EEG signal and obtain optimal reconstructed signals regarded as the most common parameter in biomedical signal processing. Author have experimented with other wavelet family as well Bior which share symmetry property and one of the subset( N) results into high correlation with this family. Detail diagram for the selection of mother wavelet is as shown below in Figure 2. For the selection of optimal mother wavelet correlation (XCorr) between the original band limited EEG signal(X) and reconstructed signal (Y) after four level of decomposition has been considered as shown in (1). The original dataset consists 100 epocs of sample size 4097 recorded for $23.6 \mathrm{sec}$

\footnotetext{
C. lassification of seizure and seizure free EEG signals using optimal mother wavelet... (Nilima Salankar)
} 
each, it is subsampled to $2300 \times 177$, thus reduced the sample size to view the details of signals more clearly. For the subset S, EEG signals of ictal activity the six wavelets have fall into highly correlated category dmey, $\mathrm{db} 7, \mathrm{db} 15, \mathrm{sym} 7$, sym15, coif5 as shown in Figure 2. dmey function has used to extract the features from subset S. For the subset N, EEG signals have been captured from seizure free time interval but from the location of tumour only two wavelets have qualified the correlation test $\mathrm{db} 4$ and bior 2.2.db4 has used for feature extraction from subset $\mathrm{N}$ as shown in Figure 2.For subset $\mathrm{F}$, EEG signals have been captured from seizure free time interval but not from the location of tumour, six wavelets have fall into highly correlated category dmey, db7, db15, sym7, sym15, coif5. dmey function has used to extract the features from subset F. For subset Z, EEG signals have been captured from healthy subjects with eyes open, five wavelets have fall into highly correlated category db7, db15, sym7, sym15, and coif5.sym7 function has used to extract the features from subset Z. For subset O, EEG signals have been captured from healthy subjects with eyes closed, six wavelets have fall into highly correlated category db7, db15, sym7, sym15, coif5 and demy. sym7 function has been used to extract the features from subset Z. After the identification of optimal mother wavelet on the basis of correlation factor, original signal have been decomposed into 4 levels as shown in Table 1.

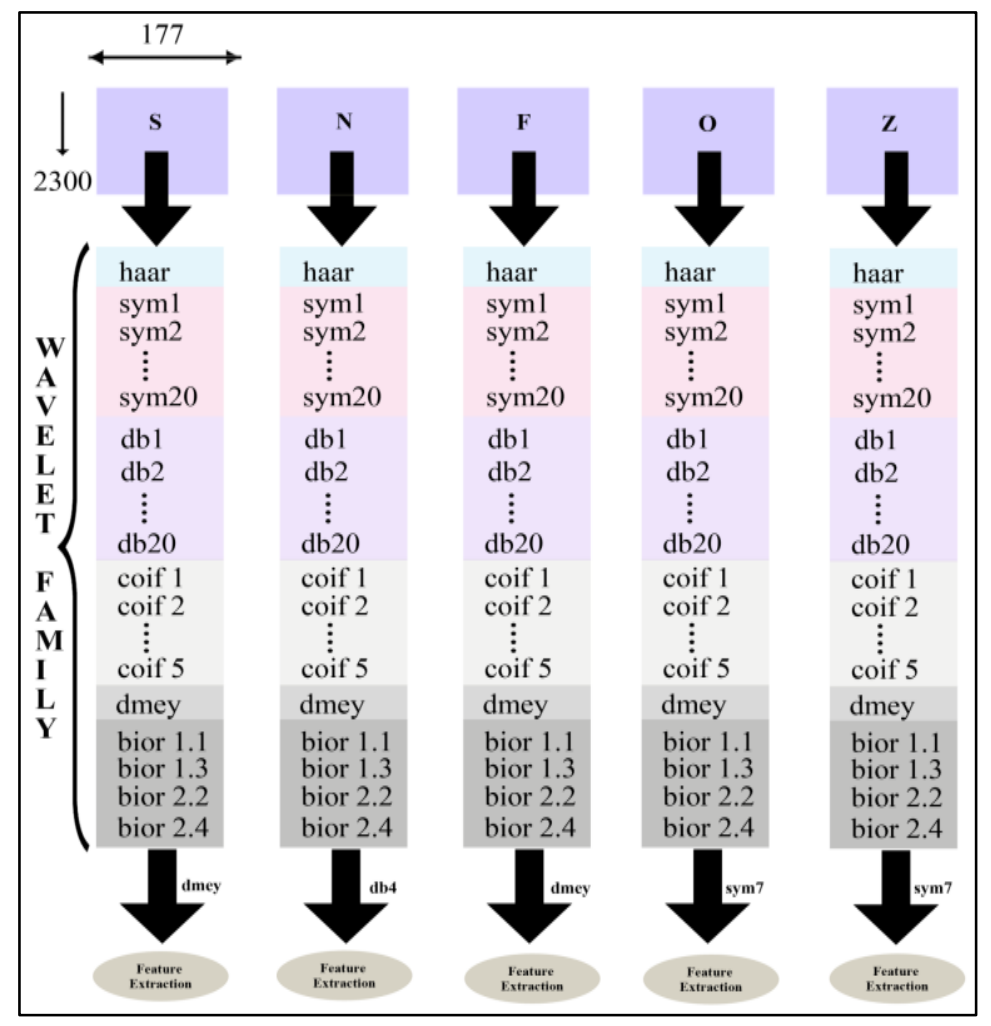

Figure 2. Optimal mother wavelet selection

Table 1. Results of four level decompostion of discrete wavelet transform

\begin{tabular}{cccc}
\hline Decomposition Level & Coefficients & Frequency Bands & Range \\
\hline 4 & A4 & $0-5.42$ & Delta \\
4 & D4 & $5.42-10.85$ & Theta(Higher) \\
3 & D3 & $10.85-21.7$ & Alpha(Higher) \\
2 & D2 & $21.7-43.40$ & Beta(Higher) \\
1 & D1 & $43.40-86.80$ & Gamma(Higher) \\
\hline
\end{tabular}

For the selection of optimal mother wavelet the correlation XCorrhas been computed between the EEG signals $(\mathrm{X})$ of range of interest and the wavelet denoised signal(Y) is expressed in (1).

$$
\operatorname{Xcorr}(\mathrm{X}, \mathrm{Y})=\frac{\sum(\mathrm{X}-\overline{\mathrm{X}})(\mathrm{Y}-\overline{\mathrm{Y}})}{\sqrt{(\mathrm{X}-\overline{\mathrm{X}})^{2}+(\mathrm{Y}-\overline{\mathrm{Y}})^{2}}}
$$

Where $\bar{X}$ and $\bar{Y}$ represents the mean values for EEG signal $\mathrm{X}$ and wavelet denoised signal $\mathrm{Y}$ respectively. 


\subsection{Feature extraction}

Feature extraction is the most important step to achieve the desired accuracy of classifier. Five features from every decomposed level has been extracted are standard deviation, kurtosis, skew, mean and relative power. Every subset Z,O, F,N and S, consists of 100 epochs of $23.6 \mathrm{sec}$ each resulted into total 4097 x 100 samples for entire duration of 23.6 x100 sec, original file has divided into the chunks of 2300 samples duration of $13.24 \mathrm{sec}$ resulting intodimension of $2300 \times 177$. Total feature values after extraction of five features from every sub band results into 177 x $25=4425$ feature values for each class. The decomposition of signal into different band are shown in Figure 3-7 for the five subsets.

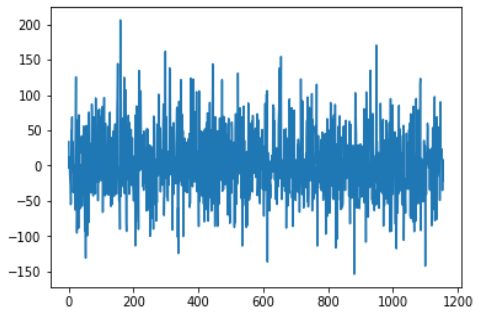

(a)

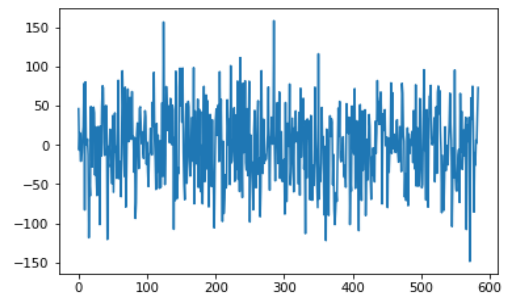

(b)

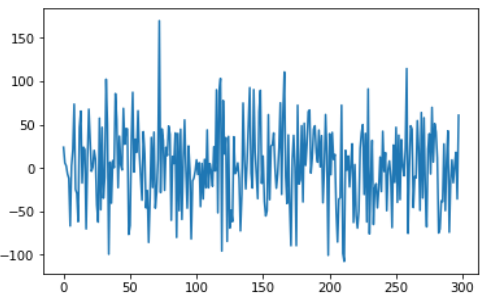

(c)

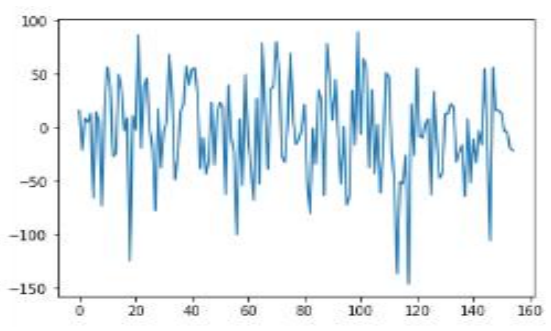

(d)

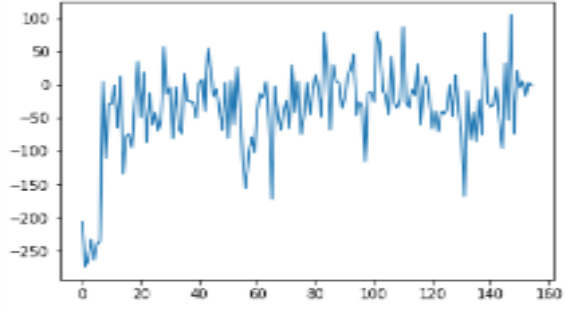

(e)

Figure 3. Decomposition of Signal from Healthy Subject with eyes open subset Z a) Detail coeffiecient of level 1 CD1. b) Detail coefficient of level 2 CD2 c)Detail coeffiecint of level 3 CD3 d) Detail coeffiecint of level 4 CD4 e) Approximate coefficient of level 4 CA4

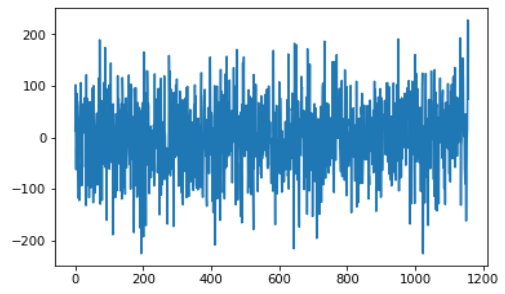

(a)

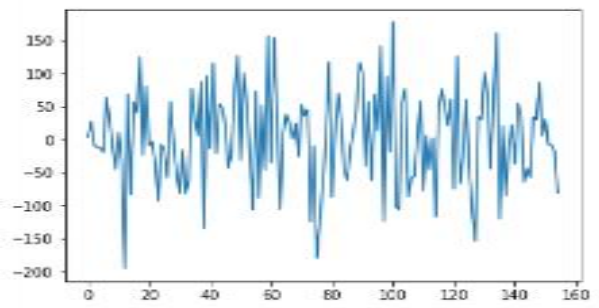

(d)

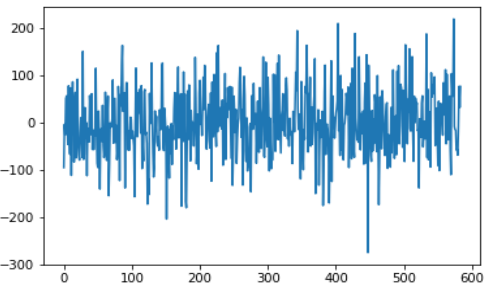

(b)

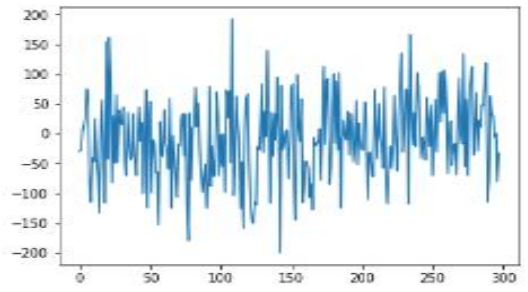

(c)

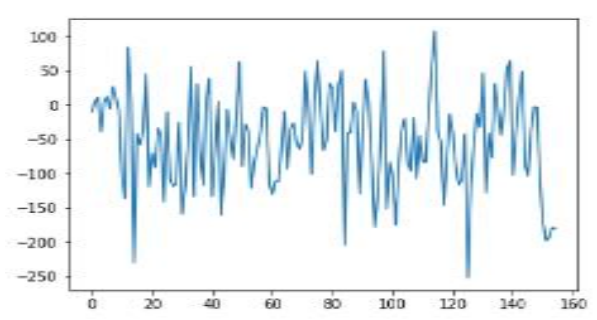

(e)

Figure 4. Decomposition of Signal from Healthy Subject with eyes close subset $\mathrm{O}$ a) Detail coeffiecient of level 1 CD1. b) Detail coefficient of level 2 CD2 c)Detail coeffiecint of level 3 CD3 d) Detail coeffiecint of level 4 CD4 e) Approximate coefficient of level 4 CA4 


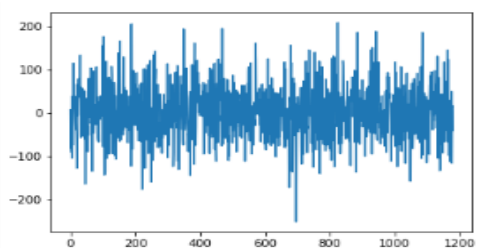

(a)

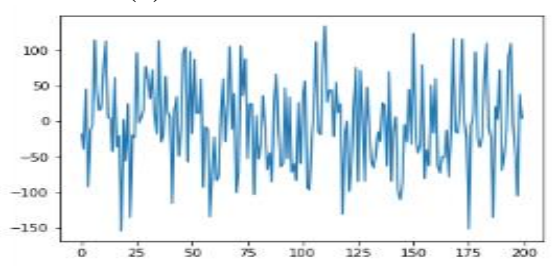

(d)

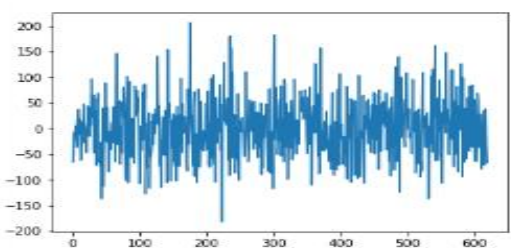

(b)

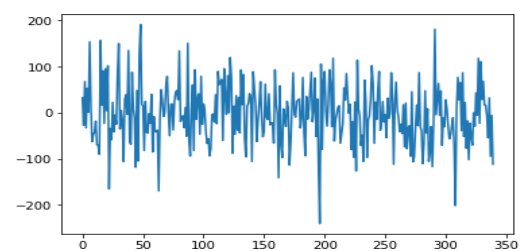

(c)

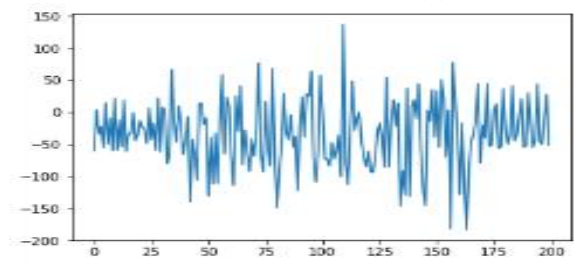

(e)

Figure 5. Decomposition of Signal from Epilepsey Subject during seizure free interval, non tumour area subset F a) Detail coeffiecient of level 1 CD1. b) Detail coefficient of level 2 CD2 c)Detail coeffiecint of level 3 CD3 d) Detail coeffiecint of level 4 CD4 e) Approximate coefficient of level 4 CA4

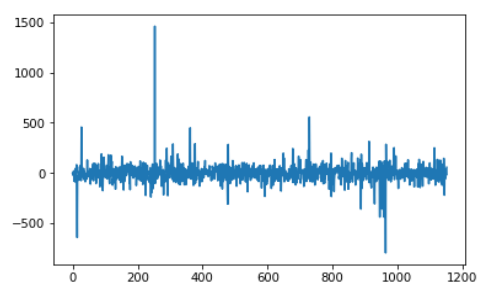

(a)

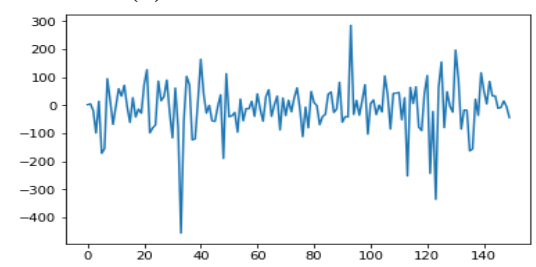

(d)

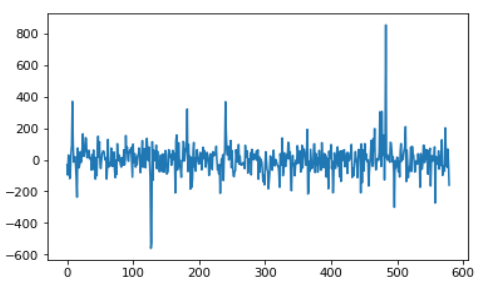

(b)

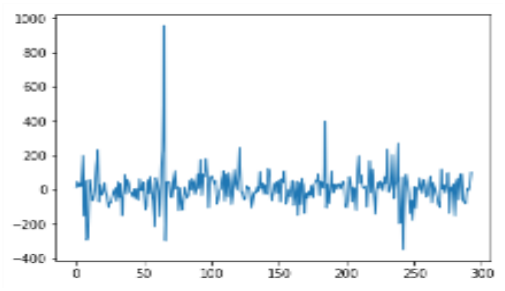

(c)

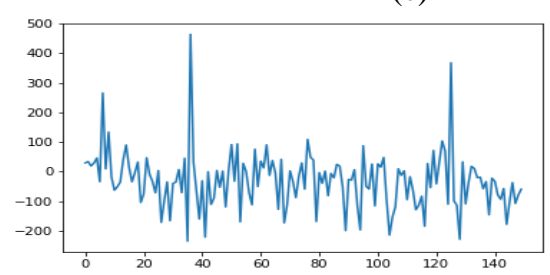

(e)

Figure 6. Decomposition of Signal from Epilepsey Subject during seizure free interval, tumour area subset N a) Detail coeffiecient of level 1 CD1. b) Detail coefficient of level 2 CD2 c)Detail coeffiecint of level 3 CD3 d) Detail coeffiecint of level 4 CD4 e) Approximate coefficient of level 4 CA4

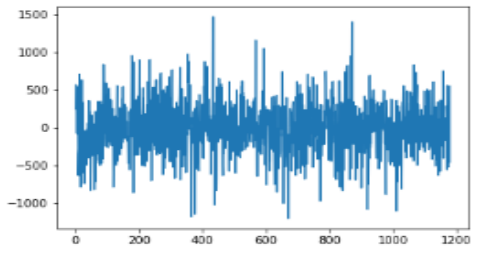

(a)

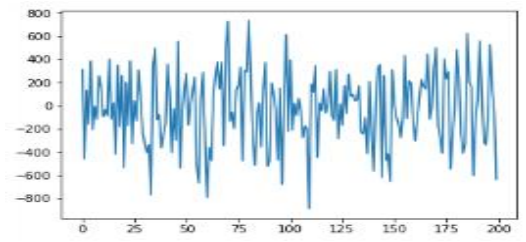

(d)

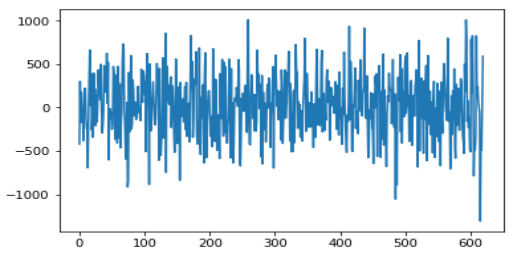

(b)

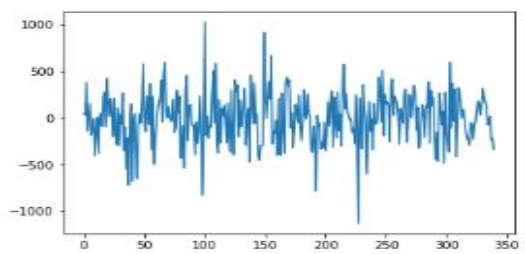

(c)

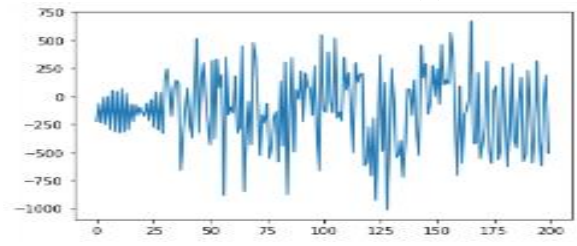

(e)

Figure 7. Decomposition of Signal from Epilepsey Subject during seizure interval, subset S a) Detail coeffiecient of level 1 CD1. b) Detail coefficient of level 2 CD2 c)Detail coeffiecint of level 3 CD3 d) Detail coeffiecint of level 4 CD4 e) Approximate coefficient of level 4 CA4 


\subsection{Design of classifier}

The entire approach used in this study from the selection of optimal mother wavelet, decomposition of EEG signals into sub bands with the help of optimal mother wavelet, feature extraction and building of classifier. In 1st step of model;Input EEG Signal--Discrete wavelet Transform and Decomposition of signal into five bands by using 51 different wavelets -reconstruction of the signal from bands - compute correlation between original signal and 51 reconstructed signals by using different wavelet--Selection of Mother wavelet on the basis of highest correlation between input signal and reconstructed signal--Optimal mother wavelet identified. In 2nd step of model;Input EEG signal-Discrete wavelet transform and decomposition of signal into five band by using optimal mother wavelet identified in step 1 of model - feature extracted from every sub band mean, standard deviation, skew, kurtosis, relative power-SVM classifier-Either Class 1 or Class 2. Classifier used in this study is of binary nature. Results are computed with linear kernel and maximum iteration limit $=100$ and numerical tolerance $=0.0010$.

\section{RESULTS}

\subsection{Statistical significance of the features}

To check for the statistical significance of total extracted 25 features at each sub band Mann Whitney $U$ test has been conducted, as this test unlike independent sample t-test does not require the feature to be normally distributed. As all the features does not satisfy the normal distribution criteria, and selected method works on the median of the feature it is assumed to be the best method to compute significance of the feature and test has conducted with the significance level $\mathrm{p}<0.05$.

\subsection{Statistical parameters for performance analysis}

The commonly used features for the performance evaluation of the classifier in case of EEG signal processing are sensitivity (SEN), specificity (SPE) and accuracy (ACC). The evaluation of the SVM classifier for the classification of Eyes Open $(Z)$ vs. Eyes Closed(O), Eyes Open $(Z) v s$. Seizure Free $(F, N)$, Eyes Open $(Z)$ vs. Seizure Free tumour free area(F), Eyes Open $(Z)$ vs. Seizure free tumour area(N), Eyes Open $(Z)$ vs. Ictal(S), Eyes Closed(O) vs. Seizure Free(F,N), Eyes Closed(O) vs. Seizure Free tumour free $\operatorname{area}(\mathrm{F})$, Eyes Closed(O) vs. Seizure free tumour area(N), Eyes Closed(O) vs. Ictal(S), Healthy(Z,O) vs. Seizure free(F,N), Seizure free Tumour free Area (F) vs. Seizure free Tumour Area(N), Seizure free Tumour free Area (F) vs. Seizure(S), Seizure free Tumor Area (N) vs. Seizure(S), Seizure(F,N) vs. Seizure (S) can be carried out by computing the SEN, SPE and ACC.In Table 2 results of performance of the classifier has enlisted and discussed in detail in discussion Section 4.

Table 2. Performance analysis of the classifier

\begin{tabular}{cccccccc}
\hline Class & SEN & SPE & ACC & Class & SEN & SPE & ACC \\
\hline Z vs. O & 99.44 & 99.42 & 99.43 & O vs. N & 99.71 & 99.71 & 99.71 \\
Z vs. FN & 100 & 100 & 100 & O vs. S & 98.87 & 98.86 & 98.87 \\
Z vs. F & 100 & 100 & 100 & ZO vs. FN & 67.37 & 78.46 & 74.64 \\
Z vs. N & 99.71 & 99.71 & 99.71 & F vs. N & 99.71 & 99.71 & 99.71 \\
Z vs. S & 100 & 100 & 100 & F vs. S & 100 & 100 & 100 \\
O vs. FN & 97.02 & 95.20 & 96.47 & N vs. S & 100 & 100 & 100 \\
O vs. F & 99.71 & 99.71 & 99.71 & FN vs. S & 99.85 & 99.71 & 99.81 \\
\hline
\end{tabular}

\section{DISCUSSION OF RESULTS}

Selection of optimal mother wavelet, experimentation has been carried out with 51 different wavelets for the healthy and non-healthy (epilepsy) subjects. Subset N (recorded EEG signals from epilepsy subjects from the location of tumor has exhibit completely different behavior from rest four subsets. Subset $\mathrm{N}$ results into high correlation with $\mathrm{Db} 4$ and Bior 2.2 which share the common property of bi-orthogonal whereas rest of the subsets doesn't fall into the range of high correlation with these two wavelets. It clearly indicates that subset $\mathrm{N}$ satisfies the bi-orthogonal property whereas rest doesn't fall into that category and hence it can lead to further base for study of epilepsy signals. For feature extraction one among the highly correlated wavelets has been selected. By assuming selected wavelet is the best choice of optimal mother wavelet for respective subset features have been extracted from each sub band delta, theta, alpha, beta and gamma. To check the significant contribution of the feature statistical significance test Mann whiteney U test has been used with significance level $\mathrm{p}<0.05$. Result signify that relative power is statistically significant for all the classification except alpha range in $\mathrm{Z}$ vs. $\mathrm{O}, \mathrm{Z}$ vs. FN and $\mathrm{Z}$ vs. F. It indicates brain activity with respect to relative power in the alpha band for this subset classification is not clearly differentiable. SD feature has exhibit random behaviour for subset to subset classification but it's clearly fails to distinguish

\footnotetext{
C. lassification of seizure and seizure free EEG signals using optimal mother wavelet... (Nilima Salankar)
} 
for the subset $\mathrm{Z}, \mathrm{O}$ vs. F,N and $\mathrm{F}$ vs. $\mathrm{N}$ for all the bands it indicates that dispersion of the data points in the dataset is almost similar and thus fail to reject the null hypothesis. Kurtosis fails to reject null hypothesis in minimum 1 band for all the subset classification but it has significantly failed in $\mathrm{Z}, \mathrm{O}$ vs. F, N and F, N vs. $\mathrm{S}$ in the subbbad beta, delta, gamma and delta, gamma theta respectively. The random behaviour appears for skew and mean as well. From the entire result analysis it's been proved that relative power stands statistically significant for the classification of EEG signals in case of seizure and seizure free. Classification of healthy+ seizure free vs. Seizure have been discarded because of unbalancing in the features which mostly reported by literature for the same dataset.The statisticalperformance evaluation results of the classifier as shown in Table 2 sensitivity lies in the range 97.02 to $100 \%$ except for healthy $(\mathrm{Z}, \mathrm{O})$ vs. seizure free activity $(\mathrm{F}, \mathrm{N})$ results into $67.37 \%$.Specificity lies in the range of $95.20 \%$ to $100 \%$ except for healthy $(\mathrm{Z}, \mathrm{O})$ vs. seizure free activity $(\mathrm{F}, \mathrm{N})$ results into 78.46 ,accuracy lies in the range of 96.47 to $100 \%$ except for healthy $(\mathrm{Z}, \mathrm{O})$ vs. seizure free activity $(\mathrm{F}, \mathrm{N})$ results into $74.64 \%$. Results indicates that merge of the recording of healthy eyes open and eyes close results into the degradation of the performance of the classifier, whereas individual recording of eyes pen vs. seizure free and eyes close vs. seizure free leads to good classification accuracy. Its again leads to explore much in this direction from eyes open and eyes close perspective.

\section{CONCLUSION}

In this paper author have explored the ability of the different wavelet families for the classification of Eyes Open $(Z)$ vs. Eyes Closed(O), Eyes Open $(Z)$ vs. Seizure Free(F,N), Eyes Open(Z) vs. Seizure Free tumour free $\operatorname{area}(\mathrm{F})$, Eyes $O$ Open(Z) vs. Seizure Free tumour $\operatorname{area}(\mathrm{N})$, Eyes $\operatorname{Open}(\mathrm{Z})$ vs. Ictal(S), Eyes Closed $(\mathrm{O})$ vs. Seizure Free(F,N), Eyes Closed(O) vs. Seizure Free tumour free area(F), Eyes Closed(O) vs. Seizure free tumour area(N), Eyes Closed(O) vs. Ictal(S), Healthy(Z,O) vs. Seizure free(F,N), Seizure free Tumour free Area $(F)$ vs. Seizure free Tumour Area(N), Seizure free Tumour free Area (F) vs. Ictal(S), Seizure free Tumour Area (N) vs. Seizure Activity (S), Seizure free (F,N) vs. Seizure activity (S). Out of the five features we have extracted from the sub bands of the EEG signal relative power stands more significant as compare to rest four features. It is necessary to test the proposed method for the classification of seizure and seizure free signals on the differentdataset to make this approach suitable for the clinical use. In future we will apply the proposed method for the classification of various other brain conditions like alzeimer.misophonia, human emotion using EEG Signals.

\section{CONFLICT OF INTEREST}

The authors declare that they do not have any conflict of interest.

\section{REFERENCES}

[1] Mormann, F., Andrzejak, R. G., Elger, C. E., \&Lehnertz, K. "Seizure prediction: the long and winding road". Brain, vol. 130, no. 2, pp. 314-333, 2006.

[2] Bethesda, M. D. "Seizures and epilepsy: Hope through research national institute of neurological disorders and stroke (ninds)", 2004. Internet Available: http://www. ninds. gov/health_and_medical/pubs/seizures_and_epilepsy_htr.htm.

[3] Porter, R. J. "The classification of epileptic seizures and epileptic syndromes". In Epilepsy: ElectroclinicalSyndromes, pp. 1-11, Springer, London, 1987.

[4] Adeli, H., Zhou, Z., \&Dadmehr, N. "Analysis of EEG records in an epileptic patient using wavelet transform", Journal of neuroscience methods, vol. 123, no. 1, pp. 69-87, 2003.

[5] Srinivasan, V., Eswaran, C., \&Sriraam, A. N. "Artificial neural network based epileptic detection using timedomain and frequency-domain features,"Journal of Medical Systems, vol. 29, no. 6, pp. 647-660, 2005.

[6] Polat, K., \&Güneş, S. "Classification of epileptiform EEG using a hybrid system based on decision tree classifier and fast Fourier transform, "Applied Mathematics and Computation,vol. 187, no. 2, pp. 1017-1026, 2007.

[7] Tzallas, A. T., Tsipouras, M. G., \& Fotiadis, D. I. "Automatic seizure detection based on time-frequency analysis and artificial neural networks, "Computational Intelligence and Neuroscience, 2007.

[8] Tzallas, A. T., Tsipouras, M. G., \& Fotiadis, D. I. "Epileptic seizure detection in EEGs using time-frequency analysis, "IEEE transactions on information technology in biomedicine, vol. 13, no. 5, pp. 703-710, 2009.

[9] Adeli, H., Zhou, Z., \&Dadmehr, N. "Analysis of EEG records in an epileptic patient using wavelet transform, Journal of neuroscience methods, vol. 123, no. 1, pp. 69-87, 2003.

[10] Khan, Y. U., \&Gotman, J. "Wavelet based automatic seizure detection in intracerebral electroencephalogram," Clinical Neurophysiology, vol. 114, no. 5, pp. 898-908, 2003.

[11] Ghosh-Dastidar, S., Adeli, H., \&Dadmehr, N. "Mixed-band wavelet-chaos-neural network methodology for epilepsy and epileptic seizure detection," IEEE transactions on biomedical engineering, vol. 54, no. 9, pp. 1545-1551, 2007. 
[12] Ocak, H. "Automatic detection of epileptic seizures in EEG using discrete wavelet transform and approximate entropy, "Expert Systems with Applications, vol. 36, no. 2, pp. 2027-2036, 2009.

[13] Adeli, H., Ghosh-Dastidar, S., \&Dadmehr, N. "A wavelet-chaos methodology for analysis of EEGs and EEG subbands to detect seizure and epilepsy, IEEE Transactions on Biomedical Engineering, vol. 54, no. 2, pp. 205-211, 2007.

[14] Subasi, A. "EEG signal classification using wavelet feature extraction and a mixture of expert model," Expert Systems with Applications, vol. 32, no. 4, pp. 1084-1093, 2007.

[15] Uthayakumar, R., \&Easwaramoorthy, D. "Epileptic seizure detection in EEG signals using multifractal analysis and wavelet transform," Fractals, vol. 21, no. 2, p. 1350011, 2013.

[16] Guo, L., Rivero, D., \&Pazos, A. "Epileptic seizure detection using multiwavelet transform based approximate entropy and artificial neural networks," Journal of neuroscience methods, vol. 193, no. 1, pp. 156-163, 2010.

[17] Steriade, M., Gloor, P. L. R. R., Llinas, R. R., Da Silva, F. L., \&Mesulam, M. M. "Basic mechanisms of cerebral rhythmic activities, Electroencephalography and clinical neurophysiology, vol 76, no. 6, pp. 481-508, 1990.

[18] Corsini, J., Shoker, L., Sanei, S., \&Alarcón, G. "Epileptic seizure predictability from scalp EEG incorporating constrained blind source separation, IEEE Transactions on biomedical Engineering, vol. 53, no. 5, pp, 790-799, 2006.

[19] Li, M., Chen, W., \& Zhang, T. “Automatic epileptic EEG detection using DT-CWT-based non-linear features," Biomedical Signal Processing and Control, vol 34, pp. 114-125, 2017.

[20] Jaiswal, A. K., \& Banka, H. "Local pattern transformation based feature extraction techniques for classification of epileptic EEG signals,” Biomedical Signal Processing and Control, vol. 34, pp. 81-92, 2017.

[21] Patidar, S., \&Panigrahi, T. "Detection of epileptic seizure using Kraskov entropy applied on tunable-Q wavelet transform of EEG signals," Biomedical Signal Processing and Control, vol. 34, pp. 74-80, 2017.

[22] Borel, C., \& Hanley, D. "Neurologic intensive care unit monitoring." Critical care clinics, vol. 1, no. 2, pp. 223-239, 1985.

[23] Agarwal, R., Gotman, J., Flanagan, D., \& Rosenblatt, B. "Automatic EEG analysis during long-term monitoring in the ICU," Electroencephalography and clinical Neurophysiology, vol. 107, no. 1, pp. 44-58, 1998.

[24] Gupta, A., Singh, P., \&Karlekar, M. "A novel signal modeling approach for classification of seizure and seizurefree EEG signals, IEEE Transactions on Neural Systems and Rehabilitation Engineering, vol. 26, no. 5, pp. 925-935, 2018.

[25] Kiranmayi, G. R., \&Udayashankara, V. "EEG subband analysis using approximate entropy for the detection of epilepsy, IOSR Journal of Computer Engineering, vol. 16, no. 5, pp. 21-27, 2014.

[26] Andrzejak, R. G., Lehnertz, K., Mormann, F., Rieke, C., David, P., \&Elger, C. E. "Indications of nonlinear deterministic and finite-dimensional structures in time series of brain electrical activity: Dependence on recording region and brain state," Physical Review E, vol. 64, no. 6, p. 061907, 2001.

\section{BIOGRAPHY OF AUTHOR}

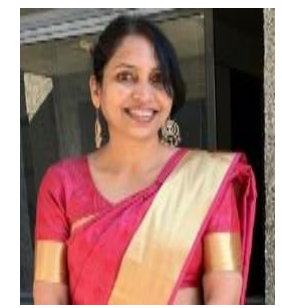

Dr. Nilima Salankar has completed her BE,Mtech and Phd in computer Science and Engineering. Her research area if interest is Human Computer Interaction,Signal Processing,Academic Research.

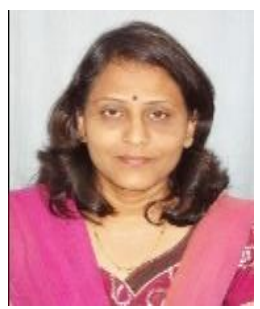

Sangita B.Nemade pursed Bachelor of Computer Science and Engineering and Master of Engineering. She is currently pursuing Ph.D in Computer Science and Engineering. Her main research area focuses on Image Processing, Machine Learning.

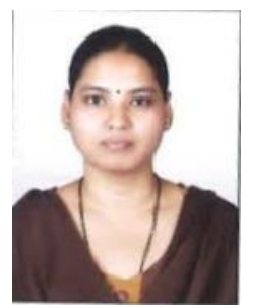

Varsha P. Gaikwad has completed her BE,ME in CSE \& pursing Phd. In Computer Sicence \& Engineering, Her Resesarch area of interest is Image processing, Remote Sensing. 